\title{
Extra-Oral Digital Panoramic Radiographic Interpretation and Clinical Findings of Stage IV Periodontitis among Some Samples Concerning Saudi/Non-Saudi Nationality
}

\author{
Mohammed M. A. Abdullah Al-Abdaly ${ }^{*}$, Amer Hassan Alasmari², Saeed J. Alqahtani², \\ Ahmed Abdulrahman Alzahrani' ${ }^{2}$, Jawaher Moshabab A. Al Ahmari², \\ Ghadeer Mohammed Mohammed Al-Abdaly3, Ibrahim Hassan Ibrahim Alqaysi², \\ Mohammed Dhafer Mohammed Al-Shamrani ${ }^{2}$ \\ ${ }^{1}$ Periodontics and Community Dental Sciences Department, College of Dentistry, King Khalid University, Abha, Saudi Arabia \\ ${ }^{2}$ College of Dentistry, King Khalid University, Abha, Saudi Arabia \\ ${ }^{3}$ Faculty of Dentistry, Sana'a University, Sana'a, Republic of Yemen \\ Email: *malabdaly20@gmail.com
}

How to cite this paper: Al-Abdaly, M.M.A.A., Alasmari, A.H., Alqahtani, S.J., Alzahrani, A.A., Al Ahmari, J.M.A., AlAbdaly, G.M.M., Alqaysi, I.H.I. and AlShamrani, M.D.M. (2022) Extra-Oral Digital Panoramic Radiographic Interpretation and Clinical Findings of Stage IV Periodontitis among Some Samples Concerning Saudi/Non-Saudi Nationality. Open Journal of Stomatology, 12, 42-61.

https://doi.org/10.4236/ojst.2022.122005

Received: January 19, 2022

Accepted: February 15, 2022

Published: February 18, 2022

Copyright $\odot 2022$ by author(s) and Scientific Research Publishing Inc. This work is licensed under the Creative Commons Attribution International License (CC BY 4.0).

http://creativecommons.org/licenses/by/4.0/

(c) (i) Open Access

\begin{abstract}
Background: There are associations between the severity of periodontal diseases and some predisposing factors, such as age, race, and income rate. Therefore, the present study was designed to evaluate the extra-oral digital panoramic radiographic interpretation and clinical findings of stage IV periodontitis among some samples concerning Saudi/non-Saudi nationality. Material and Methods: The study comprised a random sample of 298 residents in Saudi Arabia referred to the internship program clinics at the College of Dentistry, King Khalid University. They were according to the age of participants as follows: youth (15 - 24 years), adults (25 - 64 years), and seniors (65 years and over) within two groups according to nationality (Saudi and non-Saudi). Clinical periodontal examination and radiographic extra oral digital panoramic radiographs were done, and then the periodontal parameters, the percentage of bone loss, and the pattern of bone loss were recorded. The data was collected, and the statistical analysis was performed with ANOVA test, Tukey's test, Chi-square test, and T-test and considered a P-value at $(\mathrm{p}<$ $0.05)$ to be statistically significant differences and $(p<0.001)$ a high significant difference. Results: The samples included 62 (20\%) youth participants with mean of age $(21.806 \pm 3.390), 182(57 \%)$ adults' participants with mean age $(42.802 \pm 9.094)$, and $54(23 \%)$ seniors participants with a mean age $(69.074 \pm 6.810)$. There were highly statistically significant differences in the
\end{abstract}


comparison between the participants' ages $(\mathrm{P}<0.001)$. But, there were no statistically significant differences in the comparison between the nationality of participants $(\mathrm{p}=0.089)$. Adults participants had the highest score of PCR, CAL, PPD, BL, PBL, moreover, the highest scores of the clinical findings of stage IV of periodontitis when compared to youth and seniors, except \% BL and GBI where seniors participants had the highest scores $(\mathrm{p}<0.001)$. On the other hand, there were highly statistically significant differences in periodontitis grade modifiers (HbAic\% and Number of cigarettes/day) in the comparison between the participants' ages where $\mathrm{HbAic} \% \geq 7$ and Smoker $\geq 10$ cigarettes/days among the seniors participants were more than youth and adults $(\mathrm{p}<0.001)$. The results demonstrate that non-Saudi participants had the highest periodontal parameters scores moreover the highest all scores of the clinical findings of stage IV of periodontitis except the bite collapse score, tooth mobility, vertical bone loss, furcation involvement, and less than 20 remaining teeth where they were more among Saudi than non-Saudi. There were no statistically significant differences in all clinical and radiographic findings in the comparison between Saudi and non-Saudi participants except clinical attachment loss and periodontal pocket depth (PPD) ( $p>0.05)$. Conclusion: At the end of this study, we concluded that there are alterations in clinical and radiographic and clinical findings of stage IV periodontitis with the alteration of participants' ages and nationality.

\section{Keywords}

Extra-Oral Digital Panoramic Radiographs, Nationality, Stage IV

Periodontitis

\section{Introduction}

Periodontal disease is a widespread inflammatory disease in the supporting periodontal structures due to microbial dental plaque and not associated with a specific area or nationality and affecting almost the entire adult population in the world. It occurs as localized or generalized, mild, moderate or severe, gingivitis or periodontitis in particular groups [1]. It is developing due to the continuous accumulation of bacterial plaque on the tooth surfaces leading to irreversible destruction of periodontal soft tissues and alveolar bone [2]. Moreover, many studies revealed that periodontal diseases occur in all age groups, and their severity increases among older adults [3] [4] [5].

On the other hand, some racial groups of Latin and African origin are susceptible to affect severe periodontal diseases more than the other racial groups [6] [7]. This increased risk may be due to genetic factors [8]. In Saudi Arabia, there are $30.1 \%-50.8 \%$ of the population affected with periodontal diseases aged between 25 and 55 years [9].

Clinical diagnosis of periodontal diseases severity may be difficult, so clinicians should screen patients by evaluating the associated risk factors and early 
detection of periodontal diseases where the destructive periodontal disease occurs as a consequence of the reaction between host and microbial factors as well as genetic and environmental factors [10].

The orthopantomogram (A panoramic radiograph) is the fast and authoritative method that is applied for the conception and evaluation of the periodontal bone level [11]. The ratio of the periodontal bone high to the length of the root was measured to avoid elongation or shortening of the panoramic $\mathrm{x}$-ray compared with millimeters measurement in order to calculate the mean of periodontal bone loss [12].

The severe periodontal bone loss during periodontal disease may be due to irregular inflammation reaction in periodontal tissues with a microbial dental plaque with or without defect in the immune system and other predisposing factors such as the patient's age, economic status, and patient race [13].

A difference of socioeconomic factors may increase the severity of periodontitis, so the assessment of socioeconomic risk factors of dental patients, particularly with periodontal diseases, is significant in the clinical aspect that influences the patient's dental and periodontal treatment plan as well as the strategy of public health, worker training of dental care guidelines. Therefore, the objective of this study was to evaluate the extra-oral digital panoramic radiographic interpretation and clinical findings of stage IV periodontitis among some samples concerning Saudi/non-Saudi nationality.

\section{Materials and Methods}

This study was conducted at the outpatients' clinics of the internship program, college of dentistry, King Khalid University, Abha, Saudi Arabia. The ethical criteria in the 1964 announcement of Helsinki were applied during this study and the ethical clearance certificate of this study was obtained by an institutional review board (IRB), the College of Dentistry, King Khalid University (IRB/ KKUCOD/ETH/2020-21/040). Informed consent was gained from ready participants before starting the study.

Three hundred patients were screened, except two patients were not agreed to participate in the study between November 2020 to April 2021. The final sample size of this study was 298 diabetic patients with periodontal diseases were divided according to the age (Saudi and non-Saudi): Youth $(\mathrm{n}=62)$ : Range participants aged between $15-24$ ys, adults $(n=182)$ : Range participants aged between 25 - 64 ys and seniors $(n=54)$ : Range participants aged 65 years and over. The patients were categorized depending on the nationality into two groups: Saudi ( $\mathrm{n}=178)$ and non-Saudi $(\mathrm{n}=120)$.

The characteristics and Information of participants such as age, gender, nationality, past dental history, medical status, oral health status, smoking habits, and medications were recorded. The glycated hemoglobin levels (HbAlc) were obtained from the participants' medical reports.

The participants had periodontal diseases, and there was no systemic disease 
except diabetes mellitus. Thus, all participants with periodontal therapy and take antimicrobial agents, as well as the patients who have taken medication except for the drugs of diabetes within the past 6 months, were excluded. All participants had a clinical examination and full-mouth radiographic screening at internship clinics, college of dentistry, King Khalid University, Abha, Saudi Arabia.

Clinical evaluation of periodontal diseases was done. Plaque-induced gingival diseases and dental plaque were recorded, according to the gingival bleeding index (GBI) and the plaque control record (PCR) on four sites (buccal, mesial, distal, lingual) of all teeth [14] [15]. The gingival bleeding index was recorded and classified into mild $(<10 \%)$, moderate $(11 \%-30 \%)$, and severe $(>30 \%)$.

Periodontitis was evaluated using the instruction of 2017 World Workshop Periodontal diseases and conditions based on the clinical assessment of clinical attachment loss, percentage of radiographic bone loss, presence of more than 5 missing teeth due to periodontal diseases, trauma from occlusion, bite collapse, presence of less than 20 remaining teeth, the pattern of bone loss, grade of tooth mobility, and grade of furcation involvement [16].

A manual periodontal probe (UNC-15, Hu-Friedy, Chicago, IL) was used to evaluation of periodontal parameters in the present study in all teeth at six aspects (mesiobuccal, distobuccal, buccal, lingual, mesiolingual, and distolingual). Clinical attachment loss (CAL) and percentage radiographic bone loss (\%RBL) were recorded based on the following criteria: Mild (CAL $=1-2 \mathrm{~mm}$ and \%RBL $\leq 15 \%)$, moderate $(\mathrm{CAL}=3-4 \mathrm{~mm}$ and $\% \mathrm{RBL}=15 \%-33 \%)$ and severe $(\mathrm{CAL} \geq$ $5 \mathrm{~mm}$ and $\% \mathrm{RBL} \geq 30 \%$ ) [17].

The radiographic examinations of all participants were performed with extra oral digital panoramic radiographs (Planmeca Dimaxis, Planmeca Oy/Ab, Helsinki, Finland).The criteria of radiographs exclusion were based on the visibility of the alveolar bone crest (ABC), the cementoenamel junction (CEJ), and the tooth apex (AP). The radiographic bone loss was measured in the interproximal areas of teeth and recorded as a percentage for all teeth, and the mean bone loss percentage was calculated according to the following formula:

$$
\frac{(\mathrm{CEJ}-\mathrm{ABC})-2 \mathrm{~mm}}{(\mathrm{CEJ}-\mathrm{AP})-2 \mathrm{~mm}} \times 100
$$

According to the periodontal histological researches, which revealed that the distance from the crest of alveolar bone to the base of the gingival sulcus was approximately $2 \mathrm{~mm}$ (sum of the junctional epithelium $(0.97 \mathrm{~mm}$ ) and connective tissue $(1.07 \mathrm{~mm})$ attachments). Thus, There was the subtraction of $2 \mathrm{~mm}$ from distances of the $\mathrm{CEJ}$ to $\mathrm{ABC}$ and $\mathrm{CEJ}$ to $\mathrm{AP}$ as the standard in the formula in the current study [18] (Figures 1-3).

\section{Statistical Analysis}

All data were tabulated into a Microsoft Excel spreadsheet. Aone-way ANOVA test was used with Tukey test for all pairwise comparisons to calculate the mean 


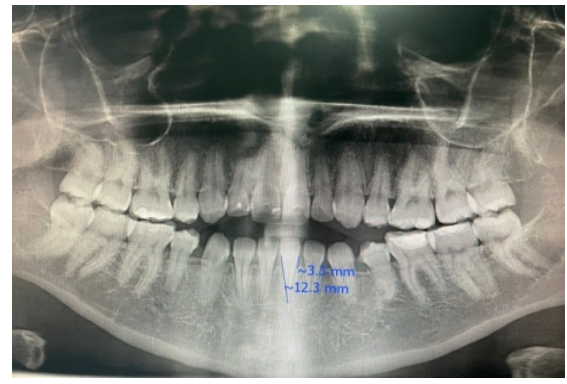

Figure 1. OPG interpretation of \#31.

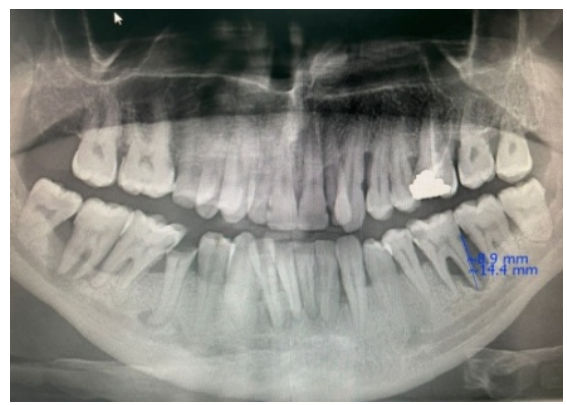

Figure 2. OPG interpretation of \#36.

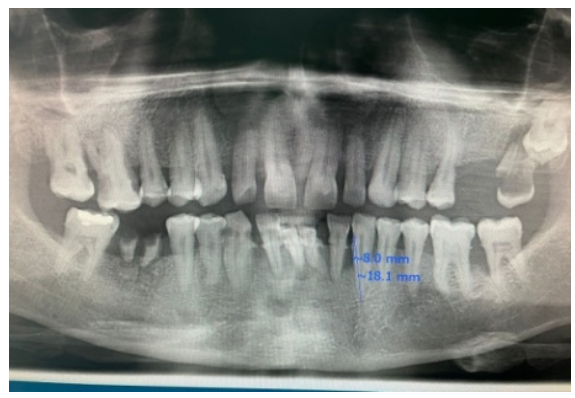

Figure 3. OPG interpretation of \#33.

and standard deviation of age, periodontal parameters, and radiographic bone loss percentage according to the study groups whereas, T-test was used to calculate the mean and standard deviation of age, periodontal parameters and radiographic bone loss percentage according to the participants' nationality. Moreover, the percentage of some participants' characteristics, clinical and radiographic findings distribution of the present study according to the participants' ages and nationality were calculated and compared using the Chi-square test. IBM SPSS Statistics 20.0.1 and Graphpad Prisms version 5 was used for all statistical tests. P-value at $<0.05$ was considered statistical significance and high statistical significance at $<0.001$.

\section{Results}

Of the 300 participants, $2(0.7 \%)$ had missing data, so they were excluded. Consequently, the remaining 298 participants included the study samples. Of these: $144(71.0 \%)$ were Saudi youth participants, 18 (29.0\%) were non-Saudi youth 
participants, $96(52.7 \%)$ were Saudi adults participants, 86 (47.3\%) were non-Saudi adults participants well as $38(70.4 \%)$ were Saudi seniors participants, and 16 (29.6\%) were non-Saudi seniors participants. The percentage of Saudi participants was more than non-Saudi participants, and there were no statistically significant differences between the participants' groups of the present study according to the nationality ( $\mathrm{p}=0.089)$.

Moreover, the percentage of controlled diabetes mellitus participants $(<7 \%$ HbA1c) (93.5\%) among youth participants was more than of adults participants (72.5\%) and seniors participants (44.4\%). In contrast, the percentage of uncontrolled diabetes mellitus participants (>7\% HbAlc) $(55.6 \%)$ among seniors participants was more than that of youth participants (6.5\%) and adults participants (27.5\%). there were highly statistically significant differences between youth, adults and seniors participants of the present study according to glycosylated hemoglobin $(\mathrm{HbA1C})$ levels $(\mathrm{p}<0.001)$. Also, Table 1 and Figure 4 revealed that the percentage of cigarettes smokers (less than $<10$ cigarettes per day) among seniors participants (92.6\%) was more than adults participants (68.2\%) and youth participants $(67.8 \%)$, whereas the percentage of cigarettes smokers (more than $<10$

Table 1. Distribution of some participants characteristics according to the age.

\begin{tabular}{|c|c|c|c|c|c|c|}
\hline \multirow{2}{*}{\multicolumn{2}{|c|}{ Participants characteristics }} & Youth & Adults & Seniors & Total & Chi-square \\
\hline & & $\mathrm{N}(\%)$ & N (\%) & $\mathrm{N}(\%)$ & N (\%) & $\mathrm{X}^{2}(\mathrm{P}$-value $)$ \\
\hline \multirow{2}{*}{$\mathrm{Na}$} & Non-Saudi & $18(29.0 \%)$ & $86(47.3 \%)$ & $16(29.6 \%)$ & $120(40.3 \%)$ & \multirow{2}{*}{$4.833(0.089)$} \\
\hline & Saudi & $44(71.0 \%)$ & $96(52.7 \%)$ & $38(70.4 \%)$ & $178(59.7 \%)$ & \\
\hline \multirow{2}{*}{$\mathrm{DM}$} & $<7 \% \mathrm{HbAlc}$ & $58(93.5 \%)$ & $132(72.5 \%)$ & $24(44.4 \%)$ & $214(71.8 \%)$ & \multirow{2}{*}{$29.243\left(<0.001^{\star}\right)$} \\
\hline & $>7 \% \mathrm{HbA} 1 \mathrm{c}$ & $4(6.5 \%)$ & $50(27.5 \%)$ & $30(55.6 \%)$ & $84(28.2 \%)$ & \\
\hline \multirow{2}{*}{$\mathrm{Sm}$} & $<10$ cig. & $42(67.8 \%)$ & $124(68.2 \%)$ & $50(92.6 \%)$ & $216(72.5 \%)$ & \multirow{2}{*}{$9.538\left(0.049^{\star}\right)$} \\
\hline & $>10$ cig. & $20(32.2 \%)$ & $58(31.8 \%)$ & $4(7.4 \%)$ & $82(27.5 \%)$ & \\
\hline
\end{tabular}

n: Number of patients, Na: Nationality, Sm: Smoking, cig: cigarettes, HbAlc: Glycated hemoglobin.

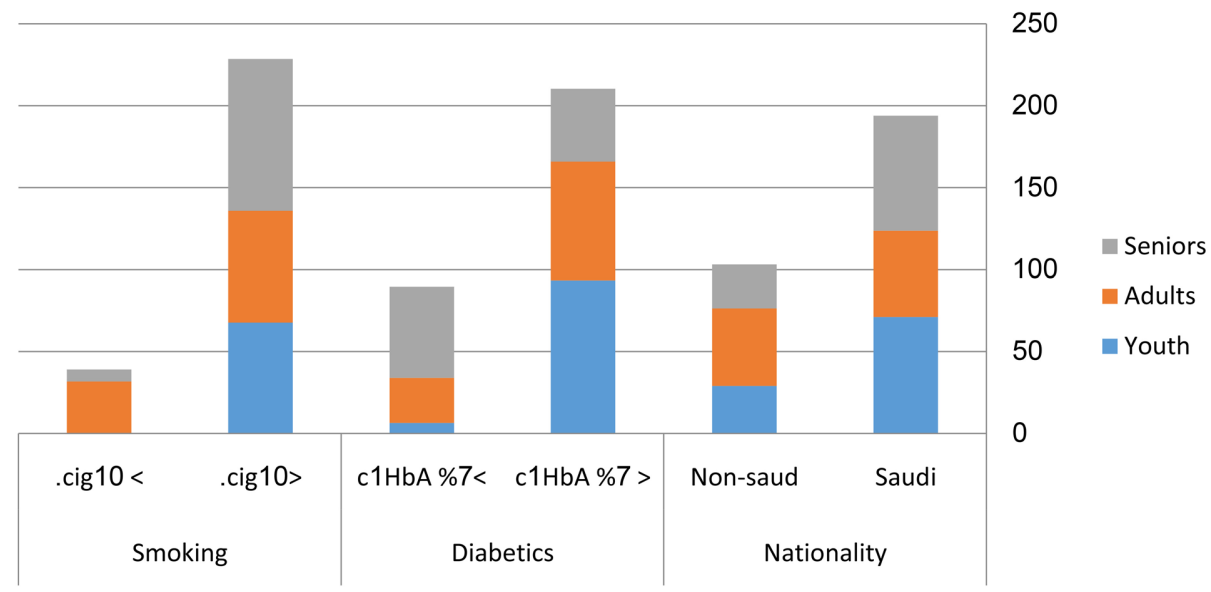

Figure 4. Participants distributions according to the age. cig: cigarettes, HbAlc: Glycated hemoglobin. 
cigarettes per day) among youth participants (32.2\%) was more than adults participants $(31.8 \%)$ and seniors participants $(7.4 \%)$. there were statistically significant differences between the groups of the present study according to glycosylated hemoglobin (HbA1C) levels and number of cigarettes per day $(<10$ cig./day and $>10$ cig./day) $(\mathrm{p}<0.05)$.

The mean and standard deviation of the different participants' ages are shown in Table 2 and Figure 5. The mean age of youth participants was 21.806 (SD: 3.390; range: 16 - 34), and the mean age of adults participants was 42.802 (SD: 9.094; range: 25 - 63) as well as the mean age of seniors participants was 69.074 (SD: 6.810; range: 60 - 95). There were highly significant differences in the mean of ages between the study participants $(\mathrm{p}<0.001)$.

Table 2, Table 3 and Figure 5, Figure 6, show the mean and standard deviation of plaque control record (PCR), gingival bleeding index (GBI), Clinical attachment loss (CAL), \% of radiographic bone loss, and periodontal pocket depth (PPD) according to the participants' ages.

The mean of CAL, \% of radiographic bone loss and PPD with standard deviation were highly statistically significantly different among the participants according to the ages $(\mathrm{p}<0.001)$, whereas there was no statistically significant difference in the mean of PCR and GBI with standard deviation among the participants according to the ages $(p>0.05)$.

Table 2. The mean of participants ages, some clinical and radiographic findings.

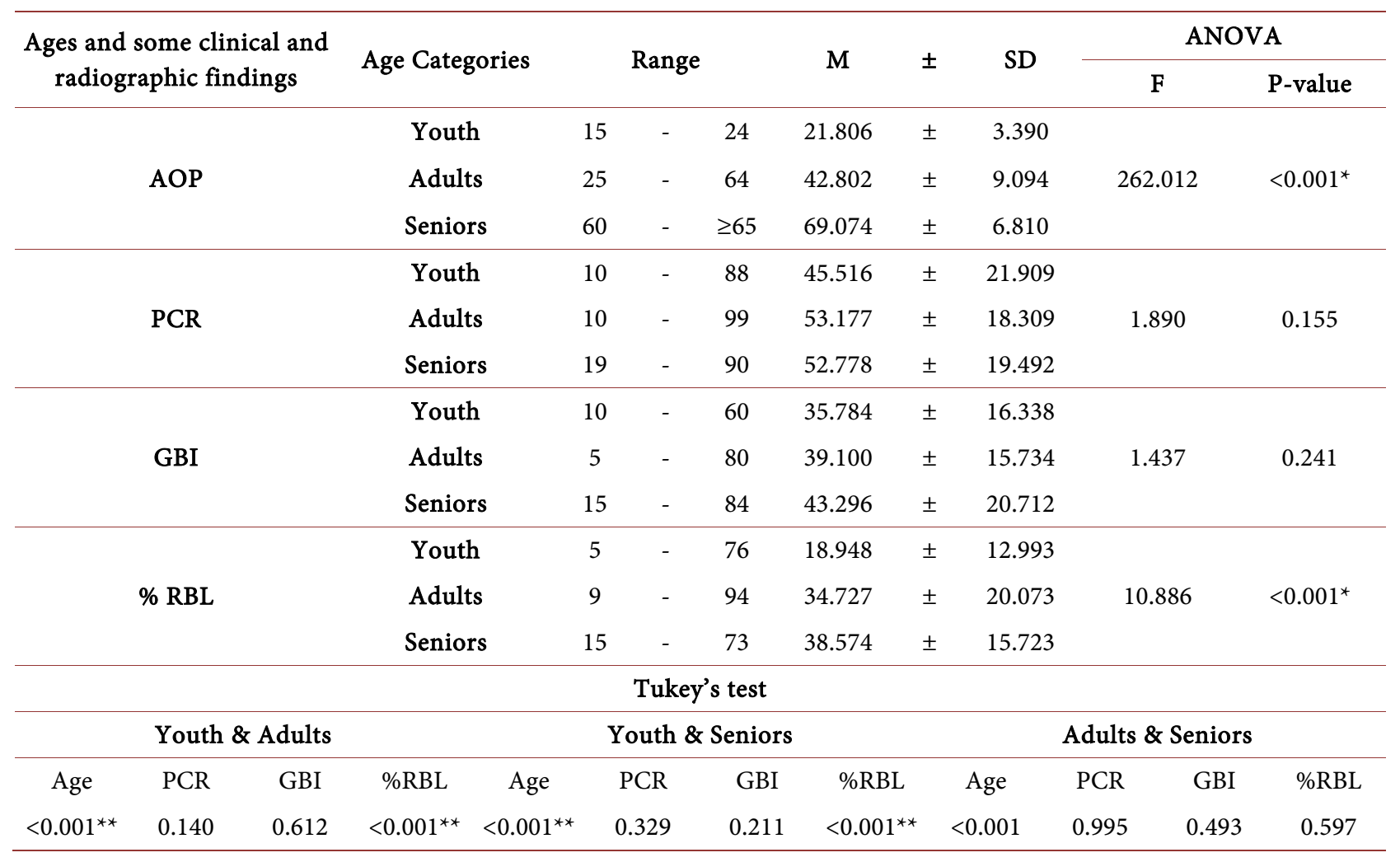

AOP: Age of patients, PCR: Plaque control record, GBI: Gingival bleeding index, \%RBL: Percentage of radiographic bone loss. ${ }^{* *}$ : Highly statistically significant differences. 


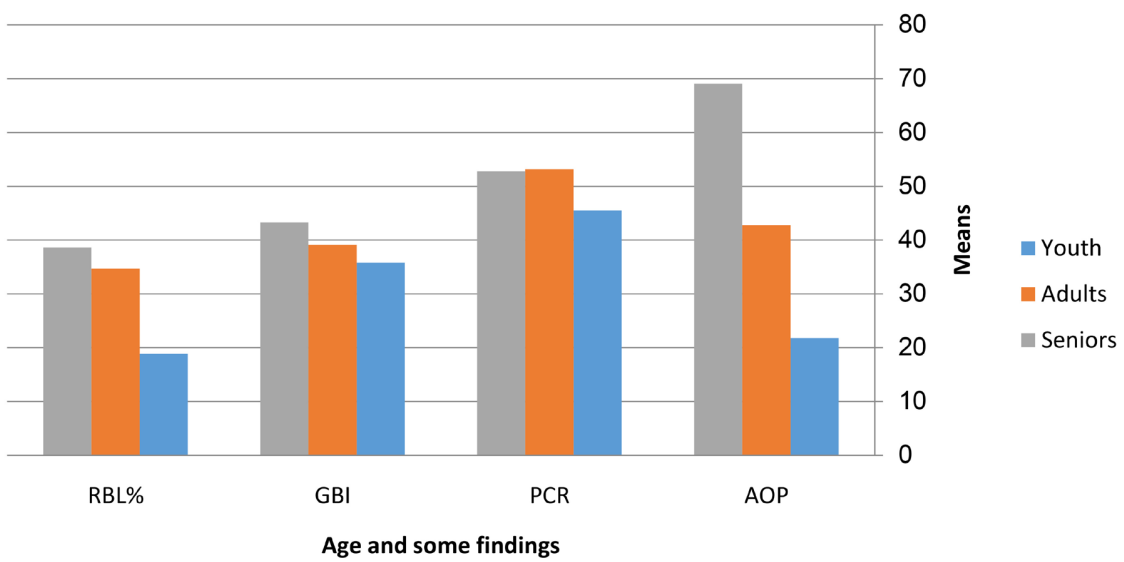

Figure 5. Mean of age and some radiographic and clinical findings. AOP: Age of patients, PCR: Plaque control record, GBI: Gingival bleeding index, \%RBL: Percentage of radiographic bone loss.

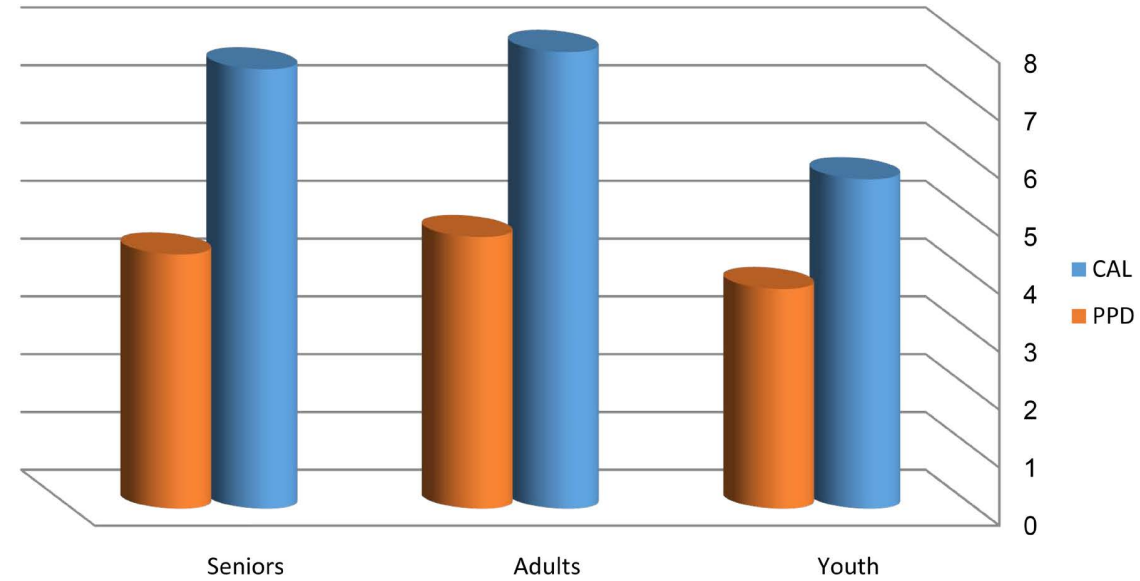

Figure 6. Mean of Clinical attachment loss and periodontal pocket depth. CAL: Clinical attachment loss, PPD: Periodontal pocket depth, CFs: Clinical findings.

Table 3. The mean of clinical attachment loss and periodontal pocket depth.

\begin{tabular}{|c|c|c|c|c|c|c|c|c|c|}
\hline \multirow{2}{*}{ CAL-PPD } & \multirow{3}{*}{$\begin{array}{c}\text { Age Categories } \\
\text { Youth }\end{array}$} & \multirow{2}{*}{\multicolumn{3}{|c|}{ Range }} & \multirow{3}{*}{$\begin{array}{c}\mathrm{M} \\
5.677\end{array}$} & \multirow{3}{*}{$\begin{array}{l} \pm \\
\pm\end{array}$} & \multirow{3}{*}{$\begin{array}{c}\text { SD } \\
1.904\end{array}$} & \multicolumn{2}{|c|}{ ANOVA } \\
\hline & & & & & & & & \multirow[t]{2}{*}{ F } & \multirow[t]{2}{*}{ P-value } \\
\hline & & 2 & - & 10 & & & & & \\
\hline \multirow[t]{3}{*}{ CAL } & Adults & 2 & - & 15 & 7.967 & \pm & 3.153 & 8.376 & $<0.001^{\star *}$ \\
\hline & Seniors & 5 & - & 12 & 7.630 & \pm & 1.573 & & \\
\hline & Youth & 2 & - & 6 & 3.806 & \pm & 1.046 & & \\
\hline \multirow[t]{2}{*}{ PPD } & Adults & 1 & - & 10 & 4.692 & \pm & 1.907 & 3.357 & $0.038^{*}$ \\
\hline & Seniors & 3 & - & 7 & 4.444 & \pm & 1.155 & & \\
\hline \multicolumn{10}{|c|}{ Tukey's test } \\
\hline \multicolumn{2}{|c|}{ Youth \& Adults } & \multicolumn{5}{|c|}{ Youth \& Seniors } & \multicolumn{3}{|c|}{ Adults \& Seniors } \\
\hline CAL & PPD & & CAL & & PPD & & CAL & & PPD \\
\hline$<0.001^{\star *}$ & $0.028^{\star}$ & & 0.019 & & 0.307 & & 0.837 & & 0.771 \\
\hline
\end{tabular}

CAL: Clinical attachment loss, PPD: Periodontal pocket depth, ${ }^{* *}$ : Highly statistically significant differences, ${ }^{*}$ : statistically significant differences. 
The mean and standard deviation of PCR (53.177 \pm 18.309), CAL (7.967 \pm 3.153), and PPD (4.692 \pm 1.907$)$ among adults participants were more than the mean and standard deviation of PCR (52.778 \pm 19.492$),(45.516 \pm 21.909)$, CAL (7.630 \pm 1.573$),(5.677 \pm 1.904)$ and PPD (4.444 \pm 1.155$),(3.806 \pm 1.046)$ among seniors participants and youth participants respectively. But the mean and standard deviation of GBI $(43.296 \pm 20.712)$ and, \% of radiographic bone loss (38.574 $\pm 15.723)$ among seniors participants were more than the mean and standard deviation of GBI $(39.100 \pm 15.734)$, ( $35.784 \pm 16.338)$, and \% of radiographic bone loss $(34.727 \pm 20.073),(18.948 \pm 12.993)$ among adults participants and youth participants respectively.

There were highly statistically significant differences $(\mathrm{p}<0.001)$ and significant differences $(\mathrm{p}<0.05)$ according Tukey's test in the comparison of youth \& adults participants (participants age, the percentage of radiographic bone loss, clinical attachment loss and periodontal pocket depth) and youth \& seniors participants (participants age, the percentage of radiographic bone loss and clinical attachment loss) as well as adults \& seniors participants (participants age).But there were no statistically significant differences according to Tukey's test in the comparison of youth \& adults participants (plaque control record and gingival bleeding index) and youth \& seniors participants (plaque control record, gingival bleeding index and periodontal pocket depth) as well as adults \& seniors participants (plaque control record, gingival bleeding index, the percentage of radiographic bone loss, clinical attachment loss and periodontal pocket depth) ( $\mathrm{p}>$ 0.05).

Table 4 and Figure 7 demonstrate there was an increase in the percentage of the affected participants with severe bone loss (63.0\%), the affected participants of 5 missing teeth and more due to periodontal diseases (66.7\%), the participants who affected with trauma from occlusion(33.3\%), the affected participants of bite collapse (33.3), less than 20 remaining teeth (10 opposing pairs) $(59.3 \%)$, the participants who affected with vertical bone loss (14.8\%), the affected participants of grade II (33.3\%) and III (37.0\%) tooth mobility as well as the participants who affected with grade II (37.0\%) and IV (33.3\%) furcation involvement among seniors participants more than the participants of adults participants and youth participants. There were highly statistically significant differences $(<0.001)$ and statistically significant differences $(\mathrm{p}<0.05)$ in the comparison between the participants clinical findings except the pattern of bone loss where there was no statistically significant difference in the comparison between the ages of participants and the pattern of bone loss $(p>0.05)$. In contrast, the results of this study exhibited that the percentage of the affected participants with mild bone loss (61.3\%) and horizontal bone loss (87.1\%) more than among adults participants and seniors participants. In addition, the percentage of adults participants was more than the percentage of seniors participants and youth participants in grade I (23.1\%) and grade III (8.8\%) furcation involvement as well as moderate bone loss $(41.8 \%)$. 
Table 4. Distribution of patients and findings according to the ages.

\begin{tabular}{|c|c|c|c|c|c|c|}
\hline \multirow{2}{*}{\multicolumn{2}{|c|}{$\begin{array}{l}\text { Clinical and } \\
\text { radiographic findings }\end{array}$}} & \multirow{2}{*}{$\begin{array}{l}\text { Youth } \\
\text { N (\%) }\end{array}$} & \multirow{2}{*}{$\begin{array}{l}\text { Adults } \\
\mathrm{N}(\%)\end{array}$} & \multirow{2}{*}{$\begin{array}{l}\text { Seniors } \\
\text { N (\%) }\end{array}$} & \multirow{2}{*}{$\begin{array}{l}\text { Total } \\
\text { N (\%) }\end{array}$} & \multirow{2}{*}{$\begin{array}{l}\text { Chi-square } \\
\mathrm{X}^{2} \text { (P-value) }\end{array}$} \\
\hline & & & & & & \\
\hline \multirow{3}{*}{$\mathrm{PeBL}$} & MI & $38(61.3 \%)$ & $46(25.3 \%)$ & $0(0.0 \%)$ & $84(28.2 \%)$ & \multirow{3}{*}{$\begin{array}{c}44.353 \\
\left(<0.001^{\star *}\right)\end{array}$} \\
\hline & MO & $22(35.5 \%)$ & $76(41.8 \%)$ & $20(37.0 \%)$ & $118(39.6 \%)$ & \\
\hline & $S$ & $2(3.2 \%)$ & $60(33.0 \%)$ & $34(63.0 \%)$ & $96(32.2 \%)$ & \\
\hline \multirow{2}{*}{$>5 \mathrm{MDP}$} & $\mathbf{N}$ & $54(87.1 \%)$ & $140(76.9 \%)$ & $18(33.3 \%)$ & $212(71.1 \%)$ & \multirow{2}{*}{$\begin{array}{c}22.533 \\
\left(<0.001^{\star *}\right)\end{array}$} \\
\hline & $\mathbf{P}$ & $8(12.9 \%)$ & $42(23.1 \%)$ & $36(66.7 \%)$ & $86(28.9 \%)$ & \\
\hline \multirow{2}{*}{ TFO } & $\mathbf{N}$ & $56(90.3 \%)$ & $124(68.1 \%)$ & $36(66.7 \%)$ & $216(72.5 \%)$ & \multirow{2}{*}{$\begin{array}{c}7.330 \\
\left(0.026^{\star}\right)\end{array}$} \\
\hline & $\mathbf{P}$ & $6(9.7 \%)$ & $58(31.9 \%)$ & $18(33.3 \%)$ & $82(27.5 \%)$ & \\
\hline \multirow{3}{*}{$\mathrm{BC}$} & $\mathbf{N}$ & $62(100.0 \%)$ & $154(84.6 \%)$ & $36(66.7 \%)$ & $252(84.6 \%)$ & \multirow{3}{*}{$\begin{array}{c}16.089 \\
\left(0.003^{\star *}\right)\end{array}$} \\
\hline & $\mathrm{D}$ & $0(0.0 \%)$ & $22(12.1 \%)$ & $12(22.2 \%)$ & $34(11.4 \%)$ & \\
\hline & F & $0(0.0 \%)$ & $6(3.3 \%)$ & $6(11.1 \%)$ & $12(4.0 \%)$ & \\
\hline \multirow{2}{*}{$<20 \mathrm{R}$} & $\mathbf{N}$ & $62(100.0 \%)$ & $164(90.1 \%)$ & $22(40.7 \%)$ & $248(83.2 \%)$ & \multirow{2}{*}{$\begin{array}{c}39.579 \\
\left(<0.001^{\star *}\right)\end{array}$} \\
\hline & $\mathbf{P}$ & $0(0.0 \%)$ & $18(9.9 \%)$ & $32(59.3 \%)$ & $50(16.8 \%)$ & \\
\hline \multirow{2}{*}{.PBL } & $\mathrm{H}$ & $54(87.1 \%)$ & $156(85.7 \%)$ & $46(85.2 \%)$ & $256(85.9 \%)$ & \multirow{2}{*}{$0.051(0.975)$} \\
\hline & $\mathrm{V}$ & $8(12.9 \%)$ & $26(14.3 \%)$ & $8(14.8 \%)$ & $42(14.1 \%)$ & \\
\hline \multirow{4}{*}{ TM } & $\mathbf{N}$ & $48(77.4 \%)$ & $82(45.1 \%)$ & $10(18.5 \%)$ & $140(47.0 \%)$ & \multirow{4}{*}{$\begin{array}{c}35.738 \\
\left(<0.001^{\star *}\right)\end{array}$} \\
\hline & Gr I & $12(19.4 \%)$ & $32(17.6 \%)$ & $6(11.1 \%)$ & $50(16.8 \%)$ & \\
\hline & Gr II & $0(0.0 \%)$ & $28(15.4 \%)$ & $18(33.3 \%)$ & $46(15.4 \%)$ & \\
\hline & GrIII & $2(3.2 \%)$ & $40(22.0 \%)$ & $20(37.0 \%)$ & $62(20.8 \%)$ & \\
\hline \multirow{5}{*}{ FI } & $\mathbf{N}$ & $56(90.3 \%)$ & $92(50.5 \%)$ & $8(14.8 \%)$ & $156(52.3 \%)$ & \multirow{5}{*}{$\begin{array}{c}62.183 \\
\left(<0.001^{\star *}\right)\end{array}$} \\
\hline & Gr I & $6(9.7 \%)$ & $42(23.1 \%)$ & $8(14.8 \%)$ & $56(18.8 \%)$ & \\
\hline & Gr II & $0(0.0 \%)$ & $24(13.2 \%)$ & $20(37.0 \%)$ & $44(14.8 \%)$ & \\
\hline & Gr III & $0(0.0 \%)$ & $16(8.8 \%)$ & $0(0.0 \%)$ & $16(5.4 \%)$ & \\
\hline & Gr IV & $0(0.0 \%)$ & $8(4.4 \%)$ & $18(33.4 \%)$ & $26(8.7 \%)$ & \\
\hline
\end{tabular}

PeBL: Periodontal bone loss, >5MDP: More than 5 missing teeth due Perio. Diseases, TFO: Trauma from occlusion, BC: Bite collapse, $<20 \mathrm{R}$ : Less than 20 remaining teeth, PBL: Pattern of bone loss, TM: Grade of tooth mobility, FI: Grade of furcation involvement, MI: Mild, MO: Moderate, S: Severe, P: Positive, N: Negative, D: Drifting, F: Flaring, H: Horizontal, V: Vertical, Gr I: Grade I, Gr II: Grade II, Gr III: Grade III, Gr IV: Grade IV, ${ }^{* *}$ : Highly statistically significant differences, ${ }^{*}$ : statistically significant differences.

Table 5 and Figure 8 exhibit the distribution of some participants characteristics according to the nationality. Overall, a higher percentage among non-Saudi more than among Saudi participants regarding uncontrolled diabetic patients (>7\% HbAlc) (33.4\%), cigarettes smokers (<10 cig./day) (78.3\%). The percentage of Saudi participants were more than among non-Saudi participants regarding controlled diabetes Mellitus (<7\% HbA1c) (75.3\%), the number of cigarettes smoked per day ( $>10$ cig./day) (33.4\%). But these differences were statistically not significant $(\mathrm{p}>0.05)$. 


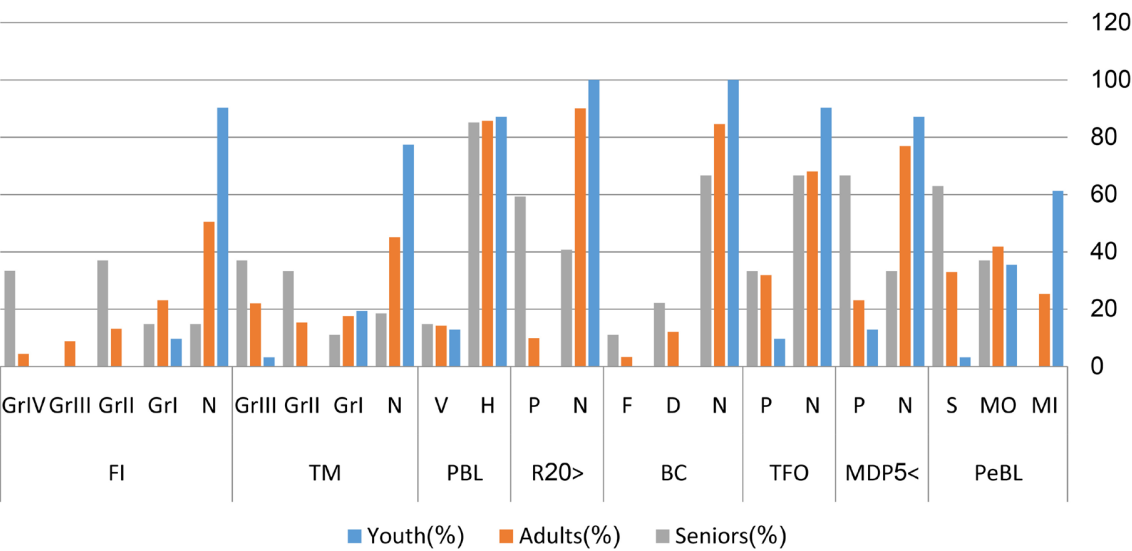

Figure 7. Distribution the participants and findings according the age. PPL: Periodontal bone loss, >5MDP: More than 5 missing teeth due Perio. Diseases, TFO: Trauma from occlusion, BC: Bite collapse, <20R: Less than 20 remaining teeth, PBL: Pattern of bone loss, TM: Grade of tooth mobility, FI: Grade of furcation involvement, MI: Mild, MO: Moderate, S: Severe, P: Positive, N: Negative, D: Drifting, F: Flaring, H: Horizontal, V: Vertical, Gr I: Grade I, Gr II: Grade II, Gr III: Grade III, Gr IV: Grade IV.

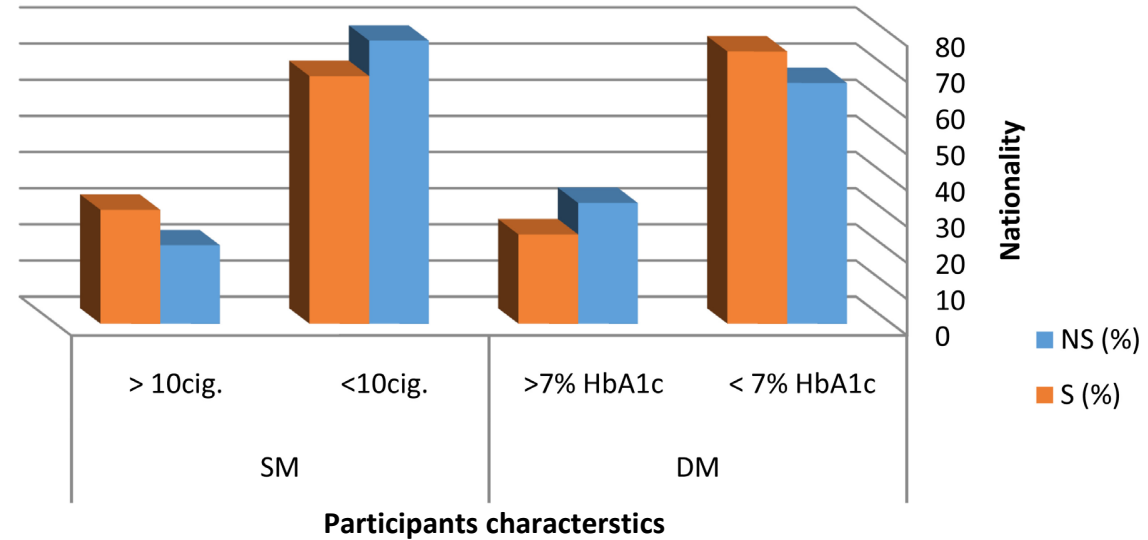

Figure 8. Participants Distribution according nationality and some characteristics. DM: Diabetes Mellitus, HbA1c: Glycated hemoglobin, Cig: Cigarettes.

Table 5. Distribution of some participants' characteristics according to the nationality.

\begin{tabular}{|c|c|c|c|c|c|}
\hline \multirow{3}{*}{\multicolumn{2}{|c|}{$\begin{array}{l}\text { Participants' } \\
\text { characteristics }\end{array}$}} & \multicolumn{3}{|c|}{ Nationality } & \multirow{3}{*}{$\begin{array}{l}\text { Chi-square } \\
\mathrm{X}^{2} \text { (P-value) }\end{array}$} \\
\hline & & \multirow{2}{*}{$\begin{array}{c}\text { Non-Saudi } \\
\mathrm{N}(\%)\end{array}$} & \multirow{2}{*}{$\begin{array}{l}\text { Saudi } \\
\text { N (\%) }\end{array}$} & \multirow{2}{*}{$\begin{array}{c}\text { Total } \\
\mathrm{N}(\%)\end{array}$} & \\
\hline & & & & & \\
\hline \multirow{2}{*}{$\mathrm{DM}$} & $<7 \%$ HbAlc & $80(66.6 \%)$ & $134(75.3 \%)$ & $214(71.8 \%)$ & \multirow{2}{*}{$1.374(0.5030)$} \\
\hline & $>7 \% \mathrm{HbA} 1 \mathrm{c}$ & $40(33.4 \%)$ & $44(24.7 \%)$ & $84(28.2 \%)$ & \\
\hline \multirow{2}{*}{ Smoking } & $<10$ cig. & $94(78.3 \%)$ & $122(68.5 \%)$ & $202(72.5 \%)$ & \multirow{2}{*}{$5.484(0.064)$} \\
\hline & $>10$ cig. & $26(21.7 \%)$ & $56(31.5 \%)$ & $82(27.5 \%)$ & \\
\hline
\end{tabular}

DM: Diabetes Mellitus, HbA1c: Glycated hemoglobin, Cig: Cigarettes.

According to Table 6 and Figure 9, the percentage of the affected participants of moderate $(45.0 \%)$ and severe $(35.0 \%)$ radiographic bone loss, the affected 
Table 6. Distribution of some participants clinical and radiographic findings according to the nationality.

\begin{tabular}{|c|c|c|c|c|c|}
\hline \multirow{3}{*}{\multicolumn{2}{|c|}{$\begin{array}{l}\text { Clinical and } \\
\text { radiographic findings }\end{array}$}} & \multicolumn{3}{|c|}{ Nationality } & \multirow{3}{*}{$\begin{array}{l}\text { Chi-square } \\
\mathrm{X}^{2} \text { (P-value) }\end{array}$} \\
\hline & & \multirow{2}{*}{$\begin{array}{c}\text { Non-Saudi } \\
\text { N (\%) }\end{array}$} & \multirow{2}{*}{$\begin{array}{l}\text { Saudi } \\
\text { N (\%) }\end{array}$} & \multirow{2}{*}{$\begin{array}{l}\text { Total } \\
\mathrm{N}(\%)\end{array}$} & \\
\hline & & & & & \\
\hline \multirow{3}{*}{ GBI } & $<10(\mathrm{MI})$ & $2(1.7 \%)$ & $2(1.1 \%)$ & $4(1.3 \%)$ & \multirow{3}{*}{$\begin{array}{c}0.837 \\
(0.658)\end{array}$} \\
\hline & $10-30(\mathrm{MO})$ & $26(21.7 \%)$ & $50(28.1 \%)$ & $76(25.5 \%)$ & \\
\hline & $>30(\mathrm{~S})$ & $92(76.7 \%)$ & $126(70.8 \%)$ & $218(73.2 \%)$ & \\
\hline \multirow{3}{*}{$\mathrm{CAL}$} & $1-2 \mathrm{~mm}(\mathrm{MI})$ & $2(1.7 \%)$ & $4(2.2 \%)$ & $6(2.0 \%)$ & \multirow{3}{*}{$\begin{array}{c}17.949 \\
\left(<0.001^{\star *}\right)\end{array}$} \\
\hline & $3-4 \mathrm{~mm}(\mathrm{MO})$ & $0(0.0 \%)$ & $32(18.0 \%)$ & $32(10.7 \%)$ & \\
\hline & $>5 \mathrm{~mm}(\mathrm{~S})$ & $118(98.3 \%)$ & $142(79.8 \%)$ & $260(87.2 \%)$ & \\
\hline \multirow{3}{*}{ Pe.BL } & MI & $24(20.0 \%)$ & $60(33.7 \%)$ & $84(28.2 \%)$ & \multirow{3}{*}{$\begin{array}{c}3.465 \\
(0.177)\end{array}$} \\
\hline & MO & $54(45.0 \%)$ & $64(36.0 \%)$ & $118(39.6 \%)$ & \\
\hline & $S$ & $42(35.0 \%)$ & $54(30.3 \%)$ & $96(32.2 \%)$ & \\
\hline \multirow{2}{*}{$>5 \mathrm{MDP}$} & $\mathrm{N}$ & $82(68.3 \%)$ & $130(73.0 \%)$ & $212(71.1 \%)$ & \multirow{2}{*}{$\begin{array}{c}0.384 \\
(0.536)\end{array}$} \\
\hline & $\mathrm{P}$ & 38 (31.7\%) & $48(27.0 \%)$ & $86(28.9 \%)$ & \\
\hline \multirow{2}{*}{ TFO } & $\mathrm{N}$ & $80(66.7 \%)$ & $136(76.4 \%)$ & $216(72.5 \%)$ & \multirow{2}{*}{$\begin{array}{c}1.687 \\
(0.194)\end{array}$} \\
\hline & $\mathrm{P}$ & $40(33.3 \%)$ & $42(23.6 \%)$ & $82(27.5 \%)$ & \\
\hline \multirow{3}{*}{ BC } & $\mathrm{N}$ & $94(78.3 \%)$ & $158(88.8 \%)$ & $252(84.6 \%)$ & \multirow{3}{*}{$\begin{array}{c}3.068 \\
(0.216)\end{array}$} \\
\hline & $\mathrm{D}$ & $20(16.7 \%)$ & $14(7.9 \%)$ & $34(11.4 \%)$ & \\
\hline & $\mathrm{F}$ & $6(5.0 \%)$ & $6(3.4 \%)$ & $12(4.0 \%)$ & \\
\hline \multirow{2}{*}{$<20 \mathrm{R}$} & $\mathrm{N}$ & $100(83.3 \%)$ & $148(83.1 \%)$ & $248(83.2 \%)$ & \multirow{2}{*}{$\begin{array}{c}0.001 \\
(0.976)\end{array}$} \\
\hline & $\mathrm{P}$ & $20(16.7 \%)$ & $30(16.9 \%)$ & $50(16.8 \%)$ & \\
\hline \multirow{2}{*}{ PBL } & $\mathrm{H}$ & $108(90.0 \%)$ & $148(83.1 \%)$ & $256(85.9 \%)$ & \multirow{2}{*}{$\begin{array}{c}1.442 \\
(0.230)\end{array}$} \\
\hline & $\mathrm{V}$ & $12(10.0 \%)$ & $30(16.9 \%)$ & $42(14.1 \%)$ & \\
\hline \multirow{4}{*}{$\mathrm{TM}$} & $\mathrm{N}$ & $46(38.3 \%)$ & $94(52.8 \%)$ & $140(47.0 \%)$ & \multirow{4}{*}{$\begin{array}{c}3.885 \\
(0.274)\end{array}$} \\
\hline & Gr I & $24(20.0 \%)$ & $26(14.6 \%)$ & $50(16.8 \%)$ & \\
\hline & Gr II & $18(15.0 \%)$ & $28(15.7 \%)$ & $46(15.4 \%)$ & \\
\hline & Gr III & $32(26.7 \%)$ & $30(16.9 \%)$ & $62(20.8 \%)$ & \\
\hline \multirow{5}{*}{ FI } & $\mathrm{N}$ & $48(40.0 \%)$ & $108(60.7 \%)$ & $156(52.3 \%)$ & \multirow{5}{*}{$\begin{array}{c}8.239 \\
(0.083)\end{array}$} \\
\hline & Gr I & $26(21.7 \%)$ & 30 (16.9\%) & $56(18.8 \%)$ & \\
\hline & Gr II & $26(21.7 \%)$ & $18(10.1 \%)$ & $44(14.8 \%)$ & \\
\hline & Gr III & $10(8.3 \%)$ & $6(3.4 \%)$ & $16(5.4 \%)$ & \\
\hline & Gr IV & $10(8.3 \%)$ & $16(9.0 \%)$ & $26(8.7 \%)$ & \\
\hline
\end{tabular}

S: Saudi, NS: Non-Saudi, GBI: Gingival bleeding Index, CAL: Clinical attachment loss, Pe.BL: Periodontal bone loss, $>5 \mathrm{MDP}$ : More than 5 missing teeth due to periodontal diseases, TFO: Trauma from occlusion, BC: Bite collapse, <20R: Less than 20 remaining teeth, PBL: Pattern of bone loss, TM: Grade of tooth mobility, FI: Grade of furcation involvement, MI: Mild, MO: Moderate, S: Severe, P: Positive, N: Negative, D: Drifting, F: Flaring, H: Horizontal, V: Vertical, Gr I: Grade I, Gr II: Grade II, Gr III: Grade III, Gr IV: Grade IV. ${ }^{* *}$ : Highly statistically significant differences. 


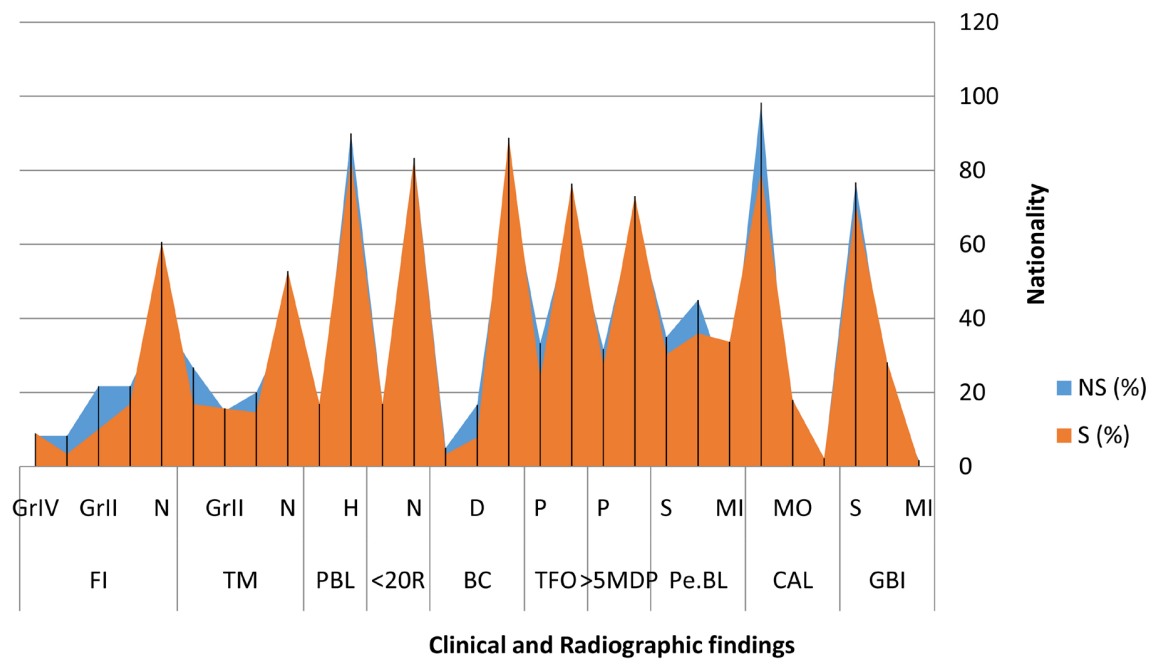

Figure 9. Clinical and radiographic findings and nationality. S: Saudi, NS: Non-Saudi, GBI: Gingival bleeding Index, CAL: Clinical attachment loss, Pe.BL: Periodontal bone loss, >5MDP: More than 5 missing teeth due to periodontal diseases, TFO: Trauma from occlusion, BC: Bite collapse, <20R: Less than 20 remaining teeth, PBL: Pattern of bone loss, TM: Grade of tooth mobility, FI: Grade of furcation involvement, MI: Mild, MO: Moderate, S: Severe, P: Positive, N: Negative, D: Drifting, F: Flaring, H: Horizontal, V: Vertical, Gr I: Grade I, Gr II: Grade II, Gr III: Grade III, Gr IV: Grade IV.

participants of 5missing teeth and more due to periodontal diseases (31.7\%), the affected participants of trauma from occlusion (33.3\%), the affected participants of bite collapse (21.7\%), the participants who affected with horizontal bone loss (90.0\%), the affected participants of grade I (20.0\%) and III (26.7\%) tooth mobility and grade I (21.7\%), II (21.7\%), III (8.3\%), and IV (8.3\%) furcation involvement, the affected participants of severe clinical attachment loss $(>5 \mathrm{~mm})$ $(98.3 \%)$ as well as the participants who affected with mild $(<10 \%)$ and severe gingival bleeding (>30\%) (76.7\%).

On the other hand, the percentage of other clinical findings and radiographic bone loss according to participants nationality among Saudi participants were more than among non-Saudi participants regarding mild bone loss (33.7\%), less than 20 remaining teeth (16.9\%), vertical bone loss (16.9\%), tooth mobility grade II (15.7\%), mild (2.2\%) and moderate (18.0\%) clinical attachment loss as well as moderate gingival bleeding (10\% - 30\%) (28.1\%). The statistically significant differences were not observed for clinical findings and radiographic bone loss according to participants' nationality $(\mathrm{p}>0.05)$ except the clinical attachment loss, where there was a highly statistically significant difference in the comparison between Saudi and non-Saudi participants $(\mathrm{p}<0.001)$.

Consequently, the results of this study displayed higher gingival and periodontal diseases and radiographic bone loss among seniors participants (65 years and over) participants more than adults participants (25- 64 years) and youth participants (15 - 24 years).

Table 7 and Figure 10 reveal the analysis of some clinical and radiographic findings (age of the participants, plaque control record, gingival bleeding index, 
Table 7. The mean and standard deviation (SD) of participants' ages, some clinical and Radiographic findings as correlated to the participants' nationality.

\begin{tabular}{|c|c|c|c|c|c|c|c|c|}
\hline & \multicolumn{6}{|c|}{ Nationality } & \multirow{2}{*}{\multicolumn{2}{|c|}{ T-test }} \\
\hline & \multicolumn{3}{|c|}{ Non-Saudi } & \multicolumn{3}{|c|}{ Saudi } & & \\
\hline & Mean & \pm & SD & Mean & \pm & SD & $\mathrm{t}$ & $P$-value \\
\hline AOP & 44.817 & \pm & 15.157 & 42.101 & \pm & 17.662 & 0.973 & 0.332 \\
\hline PCR & 54.152 & \pm & 17.486 & 49.730 & \pm & 20.538 & 1.366 & 0.174 \\
\hline GBI & 41.018 & \pm & 16.576 & 37.925 & \pm & 17.091 & 1.097 & 0.275 \\
\hline CAL & 8.233 & \pm & 2.600 & 6.888 & \pm & 2.874 & 2.911 & $0.004^{*}$ \\
\hline$\%$ RBL & 36.610 & \pm & 22.001 & 29.129 & \pm & 16.622 & 2.361 & $0.020^{*}$ \\
\hline PPD & 4.817 & \pm & 1.935 & 4.225 & \pm & 1.428 & 2.147 & $0.033^{*}$ \\
\hline
\end{tabular}

AOP: Age of patients, PCR: Plaque control record, GBI: Gingival bleeding index, \%RBL: Percentage of radiographic bone loss. *: Statistically significant differences. ${ }^{*}$ : Statistically significant differences.

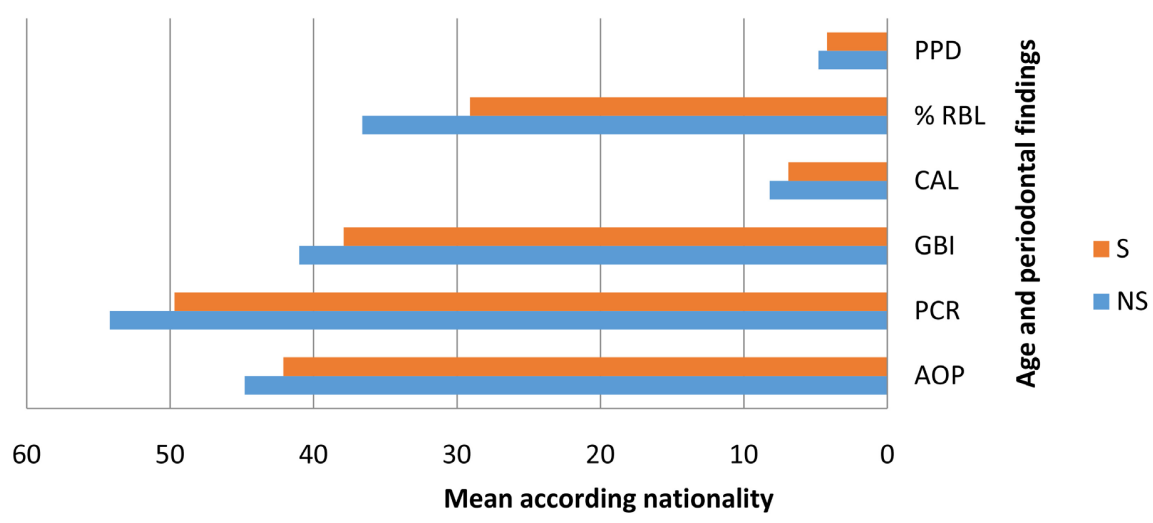

Figure 10. Participants nationality and the mean of age and periodontal findings. AOP: Age of patients, PCR: Plaque control record, GBI: Gingival bleeding index, \%RBL: Percentage of radiographic bone loss. *: Statistically significant differences. *: Statistically significant differences.

clinical attachment loss, \% of radiographic bone loss, and periodontal pocket depth) according to the participants' nationality (Saudi and non-Saudi). The comparison between Saudi and non-Saudi in revealed higher in (age of the participants, plaque control record, gingival bleeding index, clinical attachment loss, \% of radiographic bone loss, and periodontal pocket depth) among Saudi participants more than non-Saudi participants. No significant differences were showed between Saudi participants and non-Saudi participants in some clinical findings (age of the participants, plaque control record, and gingival bleeding index), but there were significant differences in other clinical and radiographic findings (clinical attachment loss, $\%$ of radiographic bone loss, and periodontal pocket depth). Overall, we found a considerable ratio of participants with gingival and periodontal diseases and radiographic bone loss among non-Saudi participants more than Saudi participants. 


\section{Discussion}

Periodontitis spread in different age groups comprising youth, adults, and seniors, which will be the anxiety of public health due to increasing of older adults and maintenance of natural teeth [19], particularly in high-income countries like Saudi Arabia. This cross-sectional study was carried out to reveal the role of the extra-oral digital panoramic radiographs as a diagnostic method in the assessment of clinical findings of stage IV periodontitis based on the presence of some secondary risk factors: age, ethnicity, diabetes mellitus and smoking on some Saudi/non-Saudi samples.

We believe that this study is from the first studies in the college of dentistry of King Khalid University aimed at extra-oral digital panoramic radiographic interpretation and clinical evaluation of the impact of patients' nationality of the residents in Saudi Arabia (Saudi and non-Saudi) on the clinical findings of stage IV periodontitis.

The study of Eke et al. in 2012 revealed that older adults have more risk of periodontal diseases than younger age groups as in this study [20]. Similar to the present study results which, revealed that seniors participants have a higher severity of radiographic bone loss and periodontal diseases compared to youth participants. These results are in agreement with many previous studies, which elucidated that the severity of periodontal diseases and the rate of periodontal bone loss increased with increased patients' ages [21] [22]. It may be attributed to the long duration of the accumulation of plaque next to the patient's periodontal tissues [23].

Moreover, these results agree with a previous study was conducted in Central Saudi Arabia, which revealed that $21 \%-32 \%$ of aged $20-64 \mathrm{yr}$ had $4-6 \mathrm{~mm}$ periodontal pocket depth as well as it demonstrated that periodontal pocket depth of $6 \mathrm{~mm}$ or greater was among of $8 \%$ of participants younger than $35 \mathrm{yr}$ old [9].

On the other hand, the severe periodontitis reaches the peak at 40 of age, and it continues as stable in older ages [24]. Similarly, the clinical finding of the present study where the clinical attachment loss among adults participants was more than youth participants and stable among seniors participants. This result coincides with the results of another study that exhibited that $18 \%$ of participants aged between 35 - 44 years had a periodontal pocket depth of $6 \mathrm{~mm}$ or more, and most of them had severe periodontitis [9]. Moreover, it conformed with the clinical findings of Lewis JM et al., and Benigeri M et al., studies [25]. [26].

A previous Saudi study revealed that plaque-induced gingivitis spread among all study participants [27]. This severity increases with diabetes mellitus (DM) which is considered one of the etiologic factors of periodontal diseases [28]. Moreover, another clinical study exhibited that the severity of periodontal bone loss increase with an increase in smoked cigarettes numbers per day [20]. These results are parallel with the results of the present study, which demonstrated that there were statistically significant differences in the clinical attachment loss and 
radiographic bone loss according to age and nationality. The above results agree with the results of Shen and his colleagues in the year 2013 study, which demonstrated that there are some factors of participants, such as residence and, income affect the periodontal health of participants. Consequently, and according to these study findings that there is an association between age and nationality of participants and the severity of periodontal diseases, and they are correlated with the results of previous studies [29].

Consequently, comparative analysis of radiographic findings with clinical periodontal parameters is an effective, helpful method for periodontal diseases diagnosis. The extra-oral digital panoramic radiographs in the present study were used to assess the severity of periodontal bone loss among Saudi/non-Saudi samples, as well as the pattern of bone loss. We found in this study that Saudi participants were affected with vertical bone loss more than among non-Saudi participants vice versa regarding the severity of radiographic bone loss where the severity of radiographic bone loss was more among non-Saudi participants compared Saudi participants. Several findings of clinical periodontal parameters and percentages of radiographic bone loss in this study were detected depending on the extra-oral digital panoramic radiographic interpretation and periodontal tissues examination.

According to the results of the current study, the non-Saudi participants' group revealed significantly clinical attachment loss $\geq 5 \mathrm{~mm}$ and periodontal pocket depth $\geq 4 \mathrm{~mm}$ as well as an increase in severity of radiographic bone loss more than the Saudi participants' group whereas, the Saudi participants group were the group showing significantly greater severity of clinical attachment loss. These differences in the severity of the periodontal disease and radiographic bone loss may be due to the alterations in the habits, behavior, and culture as well as race (genetic make-up) in the study groups. Moreover, inappropriate oral hygiene measures may be another explanation that is in agreement with the results of a previous Saudi study, which revealed that the awareness of oral health is too low among the urban population [30].

Furthermore, another Spanish study, there was an increase in gingival bleeding and tooth mobility among immigrants more than Spanish nationals [31]. These results coincide with the results of the current study where gingival bleeding and tooth mobility among non-Saudi were more than Saudi and with another Saudi study result, where there were statistically significant differences in plaque index and gingival index as well as in several parameters related to periodontitis severity among non-Saudi participants due to they do not brush their teeth. Moreover, this study results showed that brushing among Saudi participants decreased the severity of periodontitis [32].

An Egyptian previous study reported that the percentage of periodontitis severity among participants was as follow: mild (27.6\%), moderate (25.2\%), and severe (45\%) [33]. Moreover, another previous Saudi study revealed the severe periodontitis was (19.60\%) less than moderate (45.36\%) [34]. These findings 
were in agreement with Torrungruang et al., study result which demonstrated that severe periodontitis ratio is up to $15 \%$ of the participants [35]. Nevertheless, these results are in disagreement with the clinical findings of this study that demonstrated that severe periodontitis is most common among Saudi (79.8\%) and non-Saudi participants (98\%).

Generally, in this study, Non-Saudi participants were more percentage of radiographic bone loss and severe periodontal diseases compared to Saudi participants. This result agrees with multiple studies in the literature that revealed that low income is a higher risk to the severity of periodontal diseases and alveolar bone [36] [37].

\section{Strength and Limitations}

The strength of this study is to recognize the patient groups, which need higher oral health, particularly those who come from areas with low and median income. Furthermore, when we suggested conducting this study, we did not have information regarding the characteristics of these study participants looking for periodontal therapy at the college of dentistry, King Khalid University. Furthermore, the findings of this study would help to guide the general dentists and periodontists regarding diagnosis, prevention, and treatment plan of periodontal diseases among Saudi/non-Saudi most susceptible to periodontal bone loss. On the other hand, the collected and recorded data of this study permits future comparison and assessment of the stability or progression of periodontal disease among the population in Saudi Arabia. Moreover, the findings of this study will be used for evaluation of alveolar bone level in Saudi/non-Saudi samples, which may supply helpful information for periodontal treatment planning and offering more services to these groups of patients and to promote the obtain to healthcare.

The relatively small number of patients was the main limitation of this study, which could affect the accuracy of the extra-oral digital panoramic radiographic interpretation and clinical findings of periodontal status among Saudi/non-Saudi samples. Therefore, additional studies including more number of patients are highly recommended. In addition, prospective clinical studies are required to supply more explanation regarding the impact of patients' nationality on periodontal diseases severity among residents' people in Saudi Arabia. The countries of non-Saudi participants were not considered where the different cultural factors among non-Saudi participants could affect results within this group, which was another limitation in this study.

\section{Conclusions}

This study concludes that the clinical periodontal parameters of periodontal status and the extra-oral digital panoramic radiographic interpretation represent significant methods in the diagnosis of periodontal diseases severity among Saudi and non-Saudi participants. Moreover, the presence of an increase of age, as 
well as diabetes mellitus and an increase in the smoked number of cigarettes per day, increases the severity of periodontal diseases. More cross-sectional studies are recommended to exhibit the severity of periodontal diseases among Saudi/non-Saudi residents in other regions of Saudi Arabia.

In the end, periodontitis was high among non-Saudi participants compared with Saudi participants. Consequently, there was a significant association between periodontitis and the nationality of participants in the Saudi community, autonomous of other factors.

\section{Acknowledgements}

The authors introduce their thanks to the internship program committee, college of dentistry, King Khalid University, Abha, Saudi Arabia, for supporting this research work.

\section{Conflicts of Interest}

The authors reveal that there is no conflict of interest concerning this article publication.

\section{References}

[1] Burt, B. (1993) The Role of Epidemiology in the Study of Periodontal Diseases. Periodontology 2000, 2, 26-33. https://doi.org/10.1111/j.1600-0757.1993.tb00217.x

[2] Nazir, M.A. (2017) Prevalence of Periodontal Disease, Its Association with Systemic Diseases and Prevention. International Journal of Health Sciences (Qassim), 11, 72-80.

[3] Dye, B.A., Tan, S., Smith, V., Lewis, B.G., Barker, L.K., Thornton-Evans, G., et al. (2007) Trends in Oral Health Status: United States, 1988-1994 and 1999-2004. Vital and Health Statistics Series 11, No. 248, 1-92.

[4] Eke, P.I., Dye, B.A., Wei, L., Slade, G.D., Thornton-Evans, G.O., Borgnakke, W.S., et al. (2015) Update on Prevalence of Periodontitis in Adults in the United States: NHANES 2009 to 2012. Journal of Periodontology, 86, 611-622. https://doi.org/10.1902/jop.2015.140520

[5] Eke, P.I., Wei, L., Borgnakke, W.S., Thornton-Evans, G., Zhang, X., Lu, H., et al. (2016) Periodontitis Prevalence in Adults $>/=65$ Years of Age, in the USA. Periodontology 2000, 72, 76-95. https://doi.org/10.1111/prd.12145

[6] Susin, C., Haas, A.N. and Albandar, J.M. (2014) Epidemiology and Demographics of Aggressive Periodontitis. Periodontology 2000, 65, 27-45.

https://doi.org/10.1111/prd.12019

[7] Vieira, A.R. and Albandar, J.M. (2014) Role of Genetic Factors in the Pathogenesis of Aggressive Periodontitis. Periodontology 2000, 65, 92-106. https://doi.org/10.1111/prd.12021

[8] Demarco, F.F., Peres, K.G. and Peres, M.A. (2014) Life Course Epidemiology and Its Implication for Oral Health. Brazilian Oral Research, 28, 1-2. https://doi.org/10.1590/S1806-83242014.50000006

[9] Guile, E.E. (1992) Periodontal Status of Adults in Central Saudi Arabia. Community Dentistry and Oral Epidemiology, 20, 159-160. https://doi.org/10.1111/j.1600-0528.1992.tb01554.x 
[10] Kinane, D.F. (2001) Periodontal Disease in Children and Adolescents: Introduction and Classification. Periodontology2000, 26, 7-15.

https://doi.org/10.1034/j.1600-0757.2001.2260101.x

[11] Michalowicz, B., Aeppli, D., Kuba, R., Bereuter, J., Conry, J., Segal, N., Bouchard, T. and Pihlstrom, B. (1991) A Twin Study of Genetic Variation in Proportional Radiographic Alveolar Bone Height. Journal of Dental Research, 70, 1431-1435. https://doi.org/10.1177/00220345910700110701

[12] Bergström, J. and Eliasson, S. (1987) Cigarette Smoking and Alveolar Bone Height in Subjects with a High Standard of Oral Hygiene. Journal of Clinical Periodontology, 14, 466-469. https://doi.org/10.1111/j.1600-051X.1987.tb02253.x

[13] Rodrigues, M., Barbirato, D., Luiz, R.R., Scharfstein, J., Salles, G.F. and Feres-Filho, E.J. (2016) Effect of Antihypertensive Therapy with Angiotensin-Converting Enzyme Inhibitors on Chronic Periodontitis: A Case-Control Study. Oral Diseases, 22, 791-796. https://doi.org/10.1111/odi.12551

[14] Ainamo, J. and Bay, I. (1975) Problems and Proposals for Recording Gingivitis and Plaque. International Dental Journal, 25, 229-235.

[15] O’Leary, T.J., Drake, R.B. and Naylor, J.E. (1972) The Plaque Control Record. Journal of Periodontology, 43, 38. https://doi.org/10.1902/jop.1972.43.1.38

[16] Caton, J.G., Armitage, G., Berglundh, T., et al. (2018) A New Classification Scheme for Periodontal and Peri-Implant Diseases and Conditions-Introduction and Key Changes from the 1999 Classification. Journal of Periodontology, 89, S1-S8. https://doi.org/10.1002/JPER.18-0157

[17] Armitage, G.C. (1999) Development of a Classification System for Periodontal Diseases and Conditions. Annals of Periodontology, 4, 1-6. https://doi.org/10.1902/annals.1999.4.1.1

[18] Gargiulo, A.W., Wentz, F.M. and Orban, B. (1991) Dimensions and Relations of the Dentogingival Junction in Humans. Journal of Periodontology, 32, 261-267. https://doi.org/10.1902/jop.1961.32.3.261

[19] Tonetti, M.S., Bottenberg, P., Conrads, G., et al. (2017) Dental Caries and Periodontal Diseases in the Ageing Population: Call to Action to Protect and Enhance Oral Health and Well-Being as an Essential Component of Healthy Ageing-Consensus Report of Group 4 of the Joint EFP/ORCA Workshop on the Boundaries between Caries and Periodontal Diseases. Journal of Clinical Periodontology, 44, S135-S144. https://doi.org/10.1111/jcpe.12681

[20] Eke, P.I., Dye, B.A., Wei, L., Thornton-Evans, G.O. and Genco, R.J. (2012) Prevalence of Periodontitis in Adults in the United States: 2009 and 2010. Journal of Dental Research, 91, 914-920. https://doi.org/10.1177/0022034512457373

[21] Helmi, M.F., Huang, H., Goodson, J.M., et al. (2019) Prevalence of Periodontitis and Alveolar Bone Loss in a Patient Population at Harvard School of Dental Medicine. BMC Oral Health, 19, Article No. 254. https://doi.org/10.1186/s12903-019-0925-Z

[22] Papapanou, P.N. and Wennström, J.L. (1989) Radiographic and Clinical Assessments of Destructive Periodontal Disease. Journal of Clinical Periodontology, 16, 609-612. https://doi.org/10.1111/j.1600-051X.1989.tb02146.x

[23] AI Mugeiren, O.M. (2018) Assessment of Periodontal Status among the Outpatients Attending Private University Dental Clinics in Riyadh City, Saudi Arabia. Journal of International Oral Health, 10, 192-197. https://doi.org/10.4103/jioh.jioh 15218

[24] Kassebaum, N.J., Bernabé, E., Dahiya, M., Bhandari, B., Murray, C.J. and Marcenes, 
W. (2014) Global Burden of Severe Periodontitis in 1990-2010: A Systematic Review and Meta-Regression. Journal of Dental Research, 93, 1045-1053. https://doi.org/10.1177/0022034514552491

[25] Lewis, J.M., Morgan, M.V. and Clive Wright, F.A. (1994) The Validity of the CPITN Scoring and Presentation Method for Measuring Periodontal Conditions. Journal of Clinical Periodontology, 21, 1-6. https://doi.org/10.1111/j.1600-051X.1994.tb00268.x

[26] Benigeri, M., Brodeur, J.M., Payette, M., Charbonneau, A. and Ismail, A.I. (2000) Community Periodontal Index of Treatment Needs and Prevalence of Periodontal Conditions. Journal of Clinical Periodontology, 27, 308-312. https://doi.org/10.1034/j.1600-051x.2000.027005308.x

[27] Idrees, M.M., Azzeghaiby, S.N., Hammad, M.M. and Kujan, O.B. (2014) Prevalence and Severity of Plaque-Induced Gingivitis in a Saudi Adult Population. Saudi Medical Journal, 35, 1373-1377.

[28] Lamster, I.B., Lalla, E., Borgnakke, W.S. and Taylor, G.W. (2008) The Relationship between Oral Health and Diabetes Mellitus. The Journal of the American Dental Association, 139, 19S-24S. https://doi.org/10.14219/jada.archive.2008.0363

[29] Shen, J., Wildman, J. and Steele, J. (2013) Measuring and Decomposing Oral Health Inequalities in an UK Population. Community Dentistry and Oral Epidemiology, 41, 481-489. https://doi.org/10.1111/cdoe.12071

[30] Al-Otaibi, M. and Angmar-Månsson, B. (2004) Oral Hygiene Habits and Oral Health Awareness among Urban Saudi Arabians. Oral Health and Preventive Dentistry, 2, 389-396.

[31] Gómez-Costa, D., San-Roman-Montero, J., Rojo, R., et al. (2021) Self-Reported Prevalence of Periodontal Disease among the Spanish Population and Immigrants: 2006, 2011/12 and 2017: A Population-Based Study. BMC Oral Health, 21, Article No. 215. https://doi.org/10.1186/s12903-021-01579-Z

[32] AlGhamdi, A., Almarghlani, A., Alyafi, R., Ibraheem, W., Assaggaf, M., Howait, M., Alsofi, L., Banjar, A., Al-Zahrani, M. and Kayal, R. (2020) Prevalence of Periodontitis in High School Children in Saudi Arabia: A National Study. Annals of Saudi Medicine, 40, 7-14. https://doi.org/10.5144/0256-4947.2020.7

[33] Dawson, C.E. (1948) Dental Defects and Periodontal Disease in Egypt, 1946-1947. Journal of Dental Research, 27, 512-523. https://doi.org/10.1177/00220345480270041201

[34] Attia, M.A., et al. (2017) Severityof Chronic Periodontitis Associatedwith Two Major Risk Factors of PeriodontalDisease in Makah City. Journal of Dentistry and Oral Care, 3, 9-15. https://doi.org/10.15436/2379-1705.17.1316

[35] Torrungruang, K., Tamsailom, S., Rojanasomsith, K., et al. (2005) Risk Indicators of Periodontal Disease in Older Thai Adults. Journal of Periodontology, 76, 558-565. https://doi.org/10.1902/jop.2005.76.4.558

[36] Nunn, M.E. (2003) Understanding the Etiology of Periodontitis: an Overview of Periodontal Risk Factors. Periodontology 2000, 32, 11-23. https://doi.org/10.1046/j.0906-6713.2002.03202.x

[37] Elter, J.R., Beck, J.D., Slade, G.D. and Offenbacher, S. (1999) Etiologic Models for Incident Periodontal Attachment Loss in Older Adults. Journal of Clinical Periodontology, 26, 113-123. https://doi.org/10.1034/j.1600-051X.1999.260209.x 